\title{
Associações entre insatisfação corporal e frequência alimentar em adolescentes do sexo feminino
}

\author{
Associations between body dissatisfaction and food \\ frequency in female adolescents
}

Núbia de Souza de Morais ${ }^{1}$ Valter Paulo Neves Miranda', Silvia Eloiza Priore

'Universidade Federal de Viçosa

E-mail para correspondência: Núbia de Souza de Morais - nubia.s.morais25@gmail.com

\begin{abstract}
Resumo
Objetivo: Avaliar a associação da imagem corporal com a frequência alimentar em adolescentes do sexo feminino. Métodos: Estudo transversal e descritivo, com adolescentes do sexo feminino, de 14 a 19 anos, de Viçosa-MG. Medidas antropométricas foram aferidas, gordura corporal foi analisada no aparelho de bioimpedância tetrapolar, frequência alimentar (FA) foi avaliada com questionário reduzido e autoaplicável e imagem corporal foi avaliada pelo Body Shape Questionnaire (BSQ) e pela Escala de Silhuetas. Resultados: Participaram 394 adolescentes, com média de idade de 15,92 ( $\pm 1,27)$ anos, 82,4\% estavam eutróficas e 53,4\% com o percentual de gordura corporal elevado. 37,1\% tiveram a FA inadequada. Aproximadamente $50 \%$ apresentaram-se insatisfeitas com imagem corporal, pelo BSQ e Escala de Silhuetas. Adolescentes com maior insatisfação corporal faziam menos que 4 refeições nos setes dias da semana (1,55; IC95\% 1,04 - 2,32). Conclusão: Houve associação entre insatisfação corporal e diminuição da frequência de refeições das adolescentes. Este estudo confirma a importância de se investigar comportamentos de adolescentes em função da imagem corporal.
\end{abstract}

Palavras-chave: Imagem Corporal. Adolescente. Consumo alimentar.

\begin{abstract}
Objective: To evaluate the association of body image with the food frequency in female adolescents. Methods: Descriptive and cross-sectional study, with female adolescents from Viçosa-MG. Anthropometric measures were measured, body fat was analyzed in the Bio-Impedance analysis, the food frequency (FF) was evaluated using a small and self-administered questionnaire and body image was evaluated by the Body Shape Questionnaire (BSQ) and by the Silhouettes Scale. Results: A total of 394 adolescents participated in this study, with a mean age of 15.92 ( \pm 1.27) years old, $82.4 \%$ were eutrophic and $53,4 \%$ had the percentage of body fat elevated. $37.1 \%$ had inadequate FF and none of the participants reported adequate fruit consumption. Approximately $50 \%$ were dissatisfied with their body image, both by BSQ and the Silhouettes Scale. Adolescents with greater body dissatisfaction did less than 4 meals on the days of the week (1.55, 95\% Cl 7.04 - 2.32). Conclusion: There was an association between body dissatisfaction and decreased in the frequency of meals of adolescents. This study
\end{abstract}


confirms the importance of investigating the behaviors adopted by adolescents due to the evaluation of body image.

Keywords: Body Image. Adolescent. Food consumption.

\section{INTRODUÇÃO}

Adolescência é o período de transição entre infância e idade adulta, compreendendo a faixa etária de 10 a 19 anos $^{1}$. As mudanças que ocorrem nesse período influenciam o processo de formação da imagem corporal do indivíduo². Durante esse período, ocorre maior acúmulo de gordura corporal no sexo feminino, o que pode gerar insatisfação com a imagem corporal ${ }^{3}$.

Preocupações com a imagem corporal compreendem afetos negativos e cognições em relação ao próprio corpo; eles já são predominantes em meninos e meninas adolescentes e podem até aumentar durante a transição para a idade adulta jovem4. A insatisfação corporal faz parte da dimensão atitudinal desse constructo maior e representa o desejo em obter um corpo diferente de como o indivíduo o percebet. A distorção da percepção corporal se refere ao indivíduo ver seu próprio corpo de forma diferente de como realmente é, podendo subestimar ou superestimar o tamanho corporal ${ }^{4}$. As mudanças na composição corporal que ocorrem na adolescência podem levar a julgamentos da própria aparência física de forma diferente de como ele a percebe, levando ao desenvolvimento de distúrbios da imagem corporal, o que tem se tornado cada vez mais comum nessa fase da vida 5 .

Em geral, adolescentes possuem um comportamento alimentar baseado no consumo elevado de alimentos muito calóricos, ricos em gorduras, açúcares e sódio, o que contribui para o desenvolvimento de sobrepeso e obesidade ${ }^{6,7}$. A busca pelo corpo considerado ideal, sem acúmulo de gordura corporal, pode causar avaliação negativa da imagem corporal e levar a adolescente a comportamentos que prejudicam sua saúde, como a prática inadequada de exercícios físicos, uso de diuréticos e laxantes, e talvez, os recursos mais adotados sejam mudanças radicais na alimentação, como o jejum, pular refeições, vômito auto induzido e usar dietas inadequadas ${ }^{8}$. É comum observar adolescentes que realizam menos de quatro refeições por dia, e as que são mais comumente omitidas são o café da manhã, a ceia e a colação, além disso, a substituição do almoço ou jantar por lanches é normal 
nessa população ${ }^{9,10}$. Tais comportamentos podem progredir conduzindo ao desenvolvimento de transtornos alimentares, como anorexia e bulimia ${ }^{12}$.

A avaliação da imagem corporal especificamente de adolescentes do sexo feminino se justifica pelo fato de que, em geral, as meninas demonstram maior preocupação com a imagem corporal do que os meninos ${ }^{5}$. Os instrumentos validados para a análise da imagem corporal como, o Body Shape Questionnaire e as Escalas de Silhuetas, priorizam a avaliação da insatisfação com o peso e a gordura corporal, que são fatores mais observados pelas meninas no julgamento da aparência física ${ }^{8,11,12,13}$. Também, são as adolescentes que mais alteram a frequência alimentar visando a redução de peso para consequentemente se sentirem mais satisfeitas com sua imagem corporal ${ }^{8,11}$.

Assim, o objetivo deste estudo foi avaliar a associação da imagem corporal com a frequência alimentar em adolescentes do sexo feminino.

\section{MÉTODO}

Estudo transversal descritivo e analítico com adolescentes do sexo feminino, de 14 a 19 anos, residentes no município de Viçosa-MG e matriculadas na rede pública do município. A amostra foi selecionada por conglomerado. No ano de 2014, as 13 escolas do município que ofereciam ensino médio foram consultadas para informar o número de alunas com idade de 14 a 19 anos matriculadas. De acordo com essas informações, verificou-se um total de 1657 estudantes. A seleção amostral foi proporcional ao tamanho do conglomerado, com acréscimo do efeito de desenho estimado em 1,1, para corrigir a variância na amostragem utilizada para representar a população escolhida.

A partir dessa informação, o tamanho amostral foi calculado no programa StatCalc, do software EpiInfoTM, versão 7.2.0.1 (Georgia, Estados Unidos). Foram selecionadas duas escolas com o maior contingente de adolescentes do sexo feminino na faixa etária pré-determinada para a seleção da amostra.

O cálculo do tamanho amostral considerou nível de confiança de 95\%, prevalência de $35 \%$ da prevalência de insatisfação corporal em adolescentes do sexo feminino nas fases intermediária e final da adolescência ${ }^{11}$ e erro máximo admissível $5 \%$. 0 resultado apresentado para o tamanho mínimo da amostra foi de 318 indivíduos, mais o adicional de $20 \%$ para cobrir possíveis perdas; verificou-se que a quantidade recomendada de participantes foi 382 adolescentes. 
Os critérios de inclusão foram: idade (14 a 19 anos), ter apresentado menarca pelo menos há um ano, entregar o Termo de Consentimento Livre e Esclarecido (TCLE) e o Termo de Assentimento (TA) assinados pelos responsáveis e pelos próprios adolescentes, respectivamente, participar dos procedimentos de coletas, não estar participando de outra pesquisa sobre avaliação da composição corporal ou controle do estado nutricional e não estar usando algum remédio que causasse efeito psiquiátrico.

Foi apresentado à direção da escola e após o consentimento houve contato com as alunas em sala de aula para apresentação e explicação do projeto. Foram entregues o TCLE e o TA para os responsáveis e as adolescentes tomarem ciência do estudo e assinarem caso consentissem com a participação.

A participação foi voluntária, podendo a adolescente recusar-se a participar ou retirar-se do mesmo quando desejasse, sem prejuízo. O estudo foi aprovado pelo Comitê de Ética em Pesquisa com Seres Humanos da Universidade Federal de Viçosa, no ano de 2014, com número de Parecer 700.976.2014.

Após o retorno dos termos assinados, foram coletadas informações referentes a identificação das adolescentes e dados antropométricos (peso, percentual de gordura corporal (\%GC) e estatura) em espaço cedido pela escola. Nesse contato, foi avaliada a imagem corporal das adolescentes pela Escala de Silhuetas ${ }^{12}$.

Para avaliação da imagem corporal utilizou-se o Questionário de Imagem Corporal $(\mathrm{BSO})^{13}$, e a Escala de Silhuetas para Adultos Brasileiros $(\mathrm{ES})^{12}$, ambos validados para adolescentes brasileiros ${ }^{13,14}$.

O BSQ é um questionário que avalia insatisfações da imagem corporal e possui 34 itens na forma de escala Likert de pontos, que são respondidos pelo participante. A finalidade é verificar a preocupação com peso e forma corporal nos últimos 4 dias. Cada pergunta possui opções de repostas de 1 (nunca) a 6 (sempre) e de acordo com a pontuação final. Há quatro níveis de insatisfação com a aparência física $(<80$ pontos - sem insatisfação corporal; de 80 a 110 pontos - insatisfação leve; de 111 a 140 pontos - insatisfação moderada; > 140 pontos - insatisfação grave) ${ }^{13}$.

A ES é formada por 15 figuras em forma de cartões plastificados e enumerados no verso. Os valores médios de Índice de Massa Corporal (IMC) correspondentes a cada figura variam de 12,5 a 47,5 kg/m² e apresentam diferença constante de 2,5 pontos. Ela foi apresentada em ordem crescente e a voluntária primeiro escolheu a figura que 
representava melhor seu corpo atual, depois uma segunda figura, referente ao corpo que ela desejava ter.

Se a diferença entre os valores contidos no verso dos cartões escolhidos fosse entre -1 e 1 a voluntária era classificada como satisfeita. Se a figura escolhida como o corpo desejado tivesse numeração maior que a escolhida como a atual, com diferença entre estes valores maior que 1, considerava-se insatisfação positiva, ou seja, o desejo de aumentar o tamanho do corpo, e quando essa figura tivesse numeração menor, a diferença era menor que - 1 e a participante apresentava insatisfação negativa ou desejo em diminuir o tamanho do corpo ${ }^{12}$.

Com a ES avaliou-se a percepção corporal, calculada pela diferença entre o IMC da silhueta escolhida como atual e o IMC mensurado. Considerou-se distorção negativa (as meninas se viam menores do que eram) quando essa diferença foi menor que $2,49 \mathrm{~kg} / \mathrm{m}^{2}$, a diferença maior que $+2,49 \mathrm{~kg} / \mathrm{m}^{2}$ representava uma distorção positiva (se viam maiores do que eram).

A avaliação antropométrica e da composição corporal foi realizada na Divisão de Saúde da Universidade Federal de Viçosa (UFV), por uma estudante do curso de Nutrição treinada. Aferiu-se peso, estatura, percentual de gordura corporal, perímetro da cintura e dobras cutâneas. Calculou-se o Índice de Massa Corporal (IMC) e relação cintura-estatura (RCE). Para aferição do peso foi utilizada balança digital eletrônica da marca Tanita $\mathrm{BC}-543^{\circledR}$ (TMAB ${ }^{\circledR}$, Londres, Reino Unido). A estatura foi aferida utilizando-se estadiômetro portátil (Alturexata ${ }^{\circledR}$, Belo Horizonte, Brasil). O IMC foi classificado pelos percentis, de acordo com sexo e idade, utilizando as curvas de IMC/idade da $\mathrm{WHO}^{15}$.

O percentual de gordura corporal (\%GC) foi aferido utilizando-se bioimpedância Tanita $\mathrm{BC}-543^{\circledR}$ (TMAB ${ }^{\circledR}$, Londres, Reino Unido) e classificado segundo pontos de corte específicos para adolescentes do sexo feminino: $<20 \%$ baixo peso; $\geq 20$ e $<$ $25 \%$ eutrofia; $\geq 25 \%$ e $<30 \%$ risco de sobrepeso; $\geq 30 \%$ sobrepeso ${ }^{16}$.

O perímetro da cintura (PC) foi aferido no ponto médio entre a margem inferior da última costela e a crista ilíaca, no plano horizontal ${ }^{15}$, medido utilizando-se fita métrica inelástica e flexível (Cardiomed ${ }^{\circledR}$, São Luis, MA, Brasil). Foi classificado como adequado valores menores que o percentil 90 da população do estudo $(<\mathrm{P} 90)$, e elevado, valores maiores ou iguais ao percentil 90 ( $\geq$ P90). A RCE foi obtida pela relação entre a medida da cintura $(\mathrm{cm})$, e da estatura $(\mathrm{cm})$, classificada como adequada quando $<0,50 .{ }^{18}$ 
Foram aferidas as dobras cutâneas tricipital, bicipital, suprailíaca e subescapular, utilizando as técnicas propostas pela $\mathrm{WHO}^{15}$. Todas foram aferidas utilizando-se cáliper da marca Lange e foram feitas duas leituras para se utilizar a média entre os valores.

O percentual de gordura periférica foi calculado pela soma das dobras bicipital e tricipital dividido pela soma das quatro dobras multiplicado por 100. A gordura central foi calculada pela soma das dobras subescapular e suprailíaca dividida pela soma das quatro dobras multiplicado por $100^{19}$.

O questionário de frequência alimentar reduzido (QFA), aplicado por um estudante de doutorado em Ciência da Nutrição devidamente treinado, foi utilizado para avaliar o número de vezes por semana que os grupos alimentares foram consumidos. O QFA é dividido em oito grupos alimentares, são eles: frutas; hortaliças e leguminosas; tubérculos; leite e derivados; cereais, pães e massas; açúcares e doces; óleos e gorduras; e condimentos. Para análises dos dados do QFA, calculou-se as médias do número de vezes de consumo de pelo menos um alimento de cada grupo, durante sete dias ${ }^{20}$.

A classificação do QFA foi feita utilizando o método de análise de agrupamento Two Step Cluster (TSC), método não hierárquico. Para isso, os grupos alimentares foram categorizados em frequência de consumo adequado e inadequado, tendo como referência o valor específico de P75 da própria população. Assim, "frutas" (P75 = 6), "hortaliças e leguminosas" (P75 = 7), "tubérculos" (P75 = 4), "leite e derivados" (P75 = 7) e "cereais, pães e massas" (P75 = 7) foram considerados inadequados quando o número de dias relatado foi menor que P75. De modo contrário, os grupos "açúcares e doces" (P75 = 7), "óleos e gorduras" (P75 = 7) e "condimentos" (P75 = 7) foram classificados como inadequados, quando os valores foram iguais ou acima do P75.

O número de refeições diárias foi registrado com base no desjejum, colação, almoço, lanche da tarde, jantar (ou lanche) e ceia. $O$ valor médio dos sete dias foi calculado e categorizado pelo percentil $50\left(\mathrm{P}_{50}=4,0\right)$. Valores menores que $\mathrm{P}_{50}$ foram considerados baixo número de refeições.

Para categorização e agrupamento das informações da frequência alimentar utilizou-se análise de TSC. As regras para a seleção do número de grupos foram baseadas no número de aglomerados que resultou na melhor combinação de baixo 
Critério Bayesiano de Schwarz (BIC) e maior número de categorias com valores de importância próximos a 1.

O banco de dados foi elaborado no software Excel e teve dupla digitação. As análises estatísticas foram realizadas com auxílio do software Statistical Package for the Social Sciences (SPSS) for Windows, versão 17.0. Foram aplicados testes paramétricos ou não paramétricos, de acordo com a distribuição das variáveis, bem como testes de associação, correlação e análise de regressão.

O teste de normalidade de Kolmogorov-Smirnov foi utilizado para verificar a distribuição dos valores das variáveis quanto aos afastamentos observados em relação aos esperados na distribuição normal.

Para análise descritiva das variáveis foram apresentados valores de média, desviopadrão, mediana, mínimo e máximo, e frequência relativa.

Os testes do qui-quadrado de Pearson e de Fisher foram utilizados para verificar associação entre duas variáveis qualitativas. A correlação de Spearman foi usada para verificar correlação entre duas variáveis numéricas quantitativas.

O teste de regressão logística binária foi aplicado para análise da associação entre a insatisfação corporal avaliada pela Escala de Silhuetas e as variáveis de composição corporal, sendo ambas categorizadas em dois grupos para atenderem as exigências do teste. O intervalo de confiança foi de $95 \%$, para verificar o quanto uma variável da composição corporal pode influenciar na insatisfação corporal avaliada pela escala de silhuetas.

\section{RESULTADOS E DISCUSSÃO}

Participaram do estudo 394 adolescentes do sexo feminino, média de idade de 15,92 $( \pm 1,27)$ anos, sendo que $272(69,03 \%)$ tinham de 14 a 16 anos. A Tabela 1 apresenta uma análise descritiva das variáveis idade, medidas antropométricas, de composição corporal, de insatisfação e distorção corporal.

Tabela 1 - Análise descritiva das variáveis idade, medidas antropométricas e de composição corporal de adolescentes do sexo feminino de Viçosa, MG - 2014.

\begin{tabular}{lccccc}
\hline Variáveis & Média & DP & Mediana & Mínimo & Máximo \\
\hline Idade (anos) & 15,92 & 1,22 & 16,00 & 13,00 & 19,00 \\
Peso $(\mathrm{kg})$ & 57,04 & 12,49 & 54,00 & 36,00 & 125,00 \\
Estatura (cm) & 161 & 0,06 & 161 & 146 & 188 \\
IMC (kg/m 2 ) & 21,78 & 4,33 & 20,85 & 15,10 & 47,22 \\
GC $(\%)$ & 26,31 & 6,73 & 25,60 & 5,00 & 52,10 \\
\hline
\end{tabular}


Continuação da tabela 1

\begin{tabular}{lccccc}
\hline Variáveis & Média & DP & Mediana & Mínimo & Máximo \\
\hline PC (cm) & 72,27 & 9,70 & 70,50 & 30,50 & 121,00 \\
4DC & 67,30 & 25,92 & 61,50 & 29,00 & 186,00 \\
DC periférica (\%) & 11,77 & 5,75 & 10,34 & 3,79 & 46,27 \\
DC central (\%) & 20,46 & 8,78 & 18,71 & 7,21 & 50,23 \\
RCE & 0,44 & 0,05 & 0,43 & 0,19 & 0,75 \\
BSQ & 82,73 & 29,27 & 79,00 & 34,00 & 173,00 \\
IMC S.A. & 22,22 & 5,75 & 20,00 & 12,00 & 40,00 \\
Insatisfação & $-0,66$ & 2,19 & $-1,00$ & $-6,00$ & 6,00 \\
Distorção IMC & 0,58 & 3,95 & 0,06 & $-10,20$ & 12,85 \\
\hline
\end{tabular}

DP: Desvio-padrão; \%CC: Percentual de gordura corporal; PC: Perímetro da cintura; 4DC: Soma das quatro dobras cutâneas; DC: Dobra cutânea; RCE: Relação cintura-estatura; BSQ: Body Shape Questionnaire; IMC: Índice de massa corporal; IMC S.A.: IMC segundo silhueta atual.

A maioria $(82,4 \%)$ encontrava-se eutrófica de acordo com o IMC. Por outro lado, $53,4 \%$ apresentaram percentual de gordura corporal elevado.

Na classificação do QFA pela análise Two Step Cluster (TSC), classificou-se 3 grupos, categorizados como frequência alimentar (FA) adequada (31,3\%), FA moderadamente adequada (31,6\%) e FA inadequada (37,1\%). A interpretação dos grupos de acordo com a classificação adequada e inadequada da frequência do número de dias da semana dos grupos de alimentos se encontra na Tabela 2. Em nenhum dos grupos as adolescentes mostraram ter consumo regular de frutas.

Tabela 2 - Frequência de consumo dos grupos de alimentos nos 7 dias da semana pela análise da Two Step Cluster (TSC)*. Viçosa, MG - 2014.

\begin{tabular}{lccc}
\hline & \multicolumn{2}{c}{ Grupos de classificação da frequência alimentar } \\
\cline { 2 - 4 } Grupos de alimentos & $\begin{array}{c}\text { FA adequada } \\
\text { (n = 145) }\end{array}$ & $\begin{array}{c}\text { FA inadequada } \\
\text { (n = 126) }\end{array}$ & $\begin{array}{c}\text { FA } \\
\text { moderadamente } \\
\text { adequada } \\
\text { (n = 123) }\end{array}$ \\
\hline Açúcares e Doces & $100,0 \%$ adequado & $86,5 \%$ inadequado & $63,6 \%$ inadequado \\
Hortaliças & $75,3 \%$ adequado & $81,0 \%$ adequado & $90,3 \%$ inadequado \\
Leite e Derivados & $50,1 \%$ adequado & $79,3 \%$ inadequado & $87,9 \%$ inadequado \\
Óleos e Gorduras & $75,0 \%$ adequado & 73,5 inadequado & $77,4 \%$ adequado \\
Cereais, Pães e Massas & $91,9 \%$ adequado & 97,4 adequado & $58,9 \%$ adequado \\
Frutasa & $56,1 \%$ inadequado & 50,8 inadequado & $98,3 \%$ inadequado \\
Condimentos & $88,4 \%$ adequado & $60,3 \%$ adequado & $86,1 \%$ inadequado \\
Tubérculos & $74,3 \%$ inadequado & $54,0 \%$ inadequado & $79,8 \%$ inadequado \\
\hline
\end{tabular}

$N=394$. FA: Frequência Alimentar. *A classificação da frequência alimentar de cada grupo alimentar foi de acordo com o percentil 75 (adequado ou inadequado), sendo a interpretação de acordo com a prevalência da frequência do número de dias de consumo dos alimentos considerados saudáveis (leites e derivados, hortaliças, cereais pães e massas, frutas e tubérculos) e não saudáveis (açúcares e doces, óleos e gorduras e condimentos). aNenhum dos grupos gerados pela TSC demonstrou a maior prevalência de frequência de consumo regular de frutas.

Em relação à Escala de Silhueta (ES), nota-se que 50\% das meninas se mostraram insatisfeitas com a imagem corporal, sendo que 35,4\% apresentaram insatisfação 
negativa e 14,6\% insatisfação positiva. De acordo com o BSQ 46,4\% das adolescentes apresentaram algum grau de insatisfação com a imagem corporal.

As análises dos resultados mostraram associação entre o número de refeições e insatisfação corporal avaliada pelo BSQ. As adolescentes que relataram fazer menos que 4 refeições diárias durante os sete dias da semana foram classificadas com maior insatisfação corporal ( $\mathrm{p}=0,029)$ (Figura 1). Este resultado não foi constatado pela avaliação da imagem corporal utilizando a escala de silhuetas (ES).

Figura 1 - Associação entre insatisfação corporal e número de refeições diárias nos 7 dias da semana. Viçosa-MG, 2014.

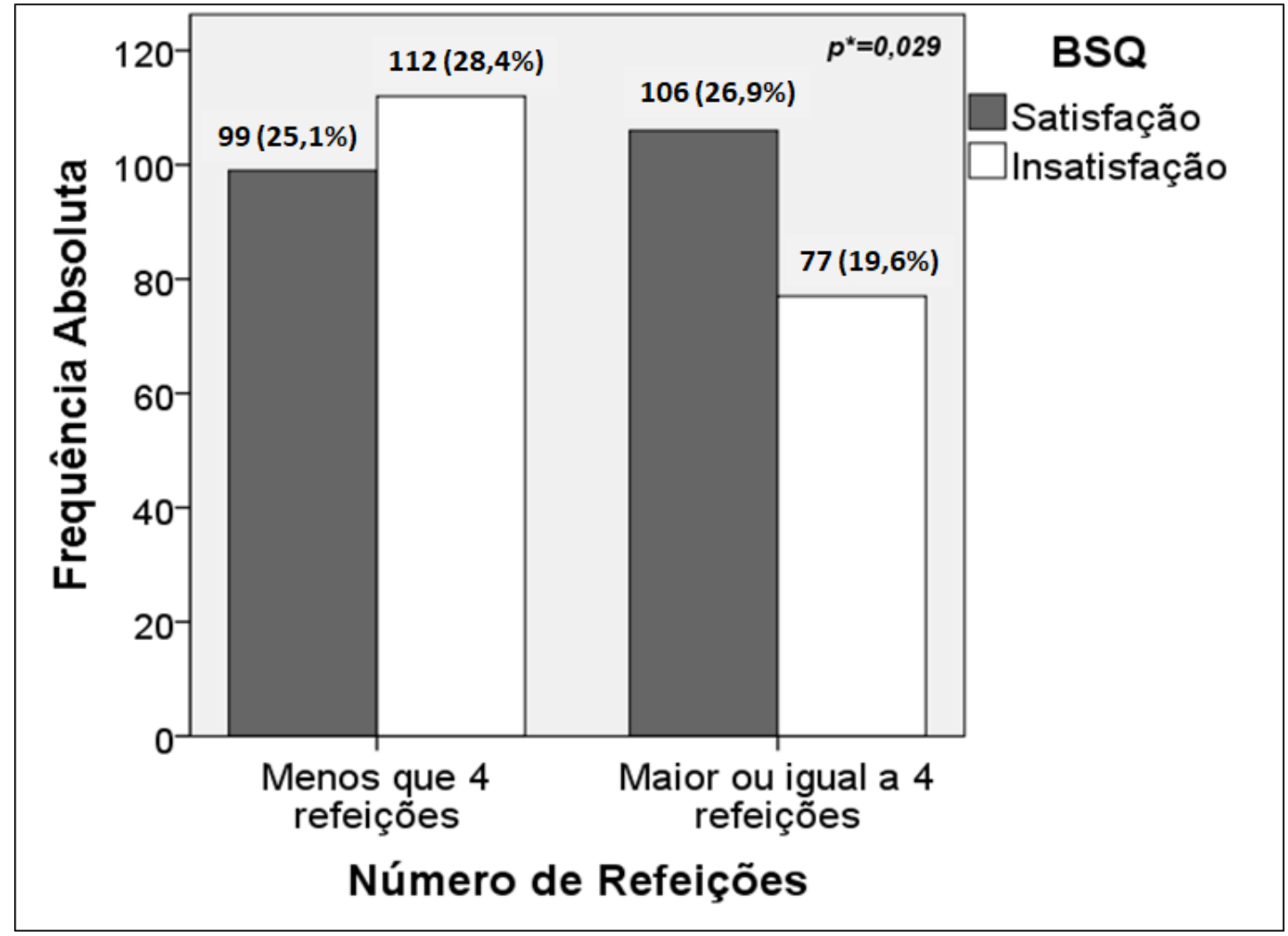

$\mathrm{N}=394 ;{ }^{*}$ Teste qui-quadrado $\left(\chi^{2}\right)$ de Pearson. BSQ: Body Shape Questionnaire

Pela análise de regressão logística simples foi possível confirmar esta associação. Observou-se que as adolescentes que relataram fazer menos que 4 refeições diárias durante os dias da semana e nos setes dias da semana, tiveram, respectivamente, 1,92 (IC95\% 1,15 - 3,20; p=0,015) e 1,55 (IC95\% 1,04 - 2,32; p=0,030) mais chances de serem classificadas como insatisfeitas pela avaliação do BSQ. Outras associações não foram encontradas envolvendo a ES e a classificação do número de refeições diárias dos sete dias da semana e no final de semana (Tabela 3). 
Tabela 3 - Classificação da imagem corporal pelo BSQ e ES em relação ao número de refeições durante a semana e no final de semana. Viçosa, MG-2014.

\begin{tabular}{|c|c|c|c|c|c|c|c|c|c|}
\hline \multirow{2}{*}{ Imagem Corporal } & \multicolumn{3}{|c|}{ Insatisfação Corporal BSQ } & \multicolumn{3}{|c|}{ Insatisfação Corporal ES } & \multicolumn{3}{|c|}{ Distorção Corporal } \\
\hline & OR & IC95\% & $p$ & OR & IC95\% & $p$ & OR & IC95\% & $p$ \\
\hline \multicolumn{10}{|l|}{ Dias da semana } \\
\hline$\geq 4$ refeições & 1 & & & 1 & & & 1 & & \\
\hline < 4 refeições & 1,92 & $1,15-3,20$ & $0,015^{*}$ & 1,43 & $0,68-1,49$ & 0,300 & 0,04 & $0,30-1,00$ & 0,50 \\
\hline \multicolumn{10}{|l|}{ Finais de semana } \\
\hline$\geq 4$ refeições & 1 & & & 1 & & & 1 & & \\
\hline < 4 refeições & 1,22 & $0,67-1,88$ & 0,800 & 1,28 & $0,79-2,22$ & 0,425 & 0,812 & $0,63-1,31$ & 0,400 \\
\hline \multicolumn{10}{|l|}{7 dias da semana } \\
\hline$\geq 4$ refeições & 1 & & & 1 & & & 1 & & \\
\hline < 4 refeições & 1,55 & $1,04-2,32$ & $0,030^{*}$ & 0,98 & $0,66-1,45$ & 0,66 & 1,02 & 0,69 & 1,52 \\
\hline
\end{tabular}

Não foi encontrada associação entre a classificação FA pela análise TSC com a imagem corporal das adolescentes, avaliada pelo BSQ $(p=0,691)$ e pela ES $(p=0,752)$.

\section{CONCLUSÃO}

As análises dos resultados mostraram que as adolescentes que apresentaram menor número de refeições durante a semana apresentaram maior insatisfação corporal. Nenhum grupo apresentou consumo adequado de frutas e $37,1 \%$ das adolescentes foram categorizadas pela análise de agrupamento (TSC) com a frequência alimentar referente aos grupos de alimentos inadequada. É comum adolescentes apresentarem uma alimentação inadequada, com elevado consumo de alimentos industrializados, ricos em açúcares, gordura e sódio e deficientes em fibras, vitaminas e minerais. ${ }^{6,7}$ Além disso, Indivíduos mais jovens, como os adolescentes, tendem a realizar menos refeições diariamente e a trocar as grandes refeições, como almoço e jantar, por lanches. $^{21,22}$

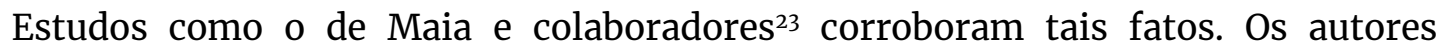
verificaram que adolescentes brasileiros de 13 a 17 anos consumiam guloseimas e alimentos salgados ultraprocessados mais de três vezes por semana. Ainda, os autores observaram que o sexo feminino se associou positivamente ao padrão alimentar não saudável (consumo de refrigerantes, guloseimas, pastéis fritos e alimentos salgados ultraprocessados). ${ }^{23}$ Ademais, Rathi, Riddell e Worsley ${ }^{24}$ observaram que adolescentes de 14 a 16 anos consumiam 6,25 porções/dia de 
lanches com alta densidade calórica. Além disso, mais da metade (59\%) não consumiam leguminosas e legumes diariamente, bem como carnes e ovos (52\%) e frutas $(45 \%)$.

A avaliação do estado nutricional mostrou que a maioria das meninas estava eutrófica, segundo o IMC, porém, 53,4\% apresentavam \%GC elevado. Estudos demonstram que essa situação é comum entre adolescentes. Faria et al. ${ }^{25}$ observaram em adolescentes do sexo feminino que a maioria era eutrófica pelo IMC, mas grande parte apresentava \%GC elevado. Resultado semelhante foi observado por Morais et al. ${ }^{26}$, em estudo realizado com 274 adolescentes brasileiras de 14 a 19 anos, no qual constataram que $15 \%$ estavam com excesso de peso pelo IMC, porém, 53,9\% apresentava excesso de gordura corporal. Ainda, entre 631 adolescentes de ambos os sexos, de 11 a 18 anos, Ripka et al. ${ }^{27}$ verificaram que o IMC superestimou o \%GC em $68,4 \%$ dos meninos $(5,0 \pm 4,0 \%)$ e subestimou em $67,5 \%$ das meninas $(-3,9 \pm$ 2,6\%). Assim, observa-se a importância de se avaliar o \%GC juntamente com o IMC para se obter uma avaliação mais completa e confiável da composição corporal, além disso, o \%GC influencia na insatisfação corporal e no comportamento alimentar de indivíduos jovens. ${ }^{28}$

As meninas na adolescência, comparadas aos meninos, podem ter maior ganho de peso e maior acúmulo de tecido adiposo, o que ocorre fisiologicamente para favorecer as alterações hormonais que ocorrem nessa fase. ${ }^{3}$ Fatores socioculturais exercem forte pressão na população referente ao padrão de beleza com excessiva valorização do corpo magro, influenciando na percepção da própria imagem, levando à insatisfação com a imagem corporal ${ }^{2,29}$.

As mulheres são mais insatisfeitas, por influências de fatores psicológicos e socioculturais, já que muitas vezes recebem estímulos externos (de mídias, amigos, entre outros) para praticarem atividades físicas e adotarem dietas para perda de peso, sem buscar orientações de profissionais qualificados. ${ }^{2}$ Estudos mostram que a maior prevalência de transtornos alimentares ocorre em indivíduos do sexo feminino, sendo assim, pesquisas que avaliem o comportamento alimentar e insatisfação corporal nesse gênero são de grande importância ${ }^{13,29}$.

A insatisfação corporal avaliada pela Escala de Silhuetas (ES) foi observada em $50 \%$ das adolescentes, sendo que entre essas, 35,4\% apresentaram insatisfação negativa, ou seja, desejo em diminuir a silhueta. Alvarenga et al. ${ }^{30}$ avaliaram insatisfação corporal de estudantes universitárias pela ES, os autores também verificaram que a 
maior parte delas $(64,4 \%)$ desejava uma silhueta menor que a atual. Os autores afirmam que a pressão que os indivíduos sofrem para se atingir o corpo considerado ideal, leva ao desejo de se obter um corpo magro, uma vez que esse objetivo não é alcançado, ocorre a insatisfação com a própria aparência física.

A percepção da imagem corporal foi avaliada pela ES e foi observado que as adolescentes não apresentaram distorção, mostrando que houve semelhança entre os valores de IMC avaliados pela Escala com os valores de IMC aferidos. Dessa forma, esse pode ser um bom instrumento para se estimar o IMC real de indivíduos.

Pela avaliação do BSQ observou-se que 45,5\% das adolescentes apresentaram algum grau de insatisfação corporal. Miranda et al. ${ }^{17}$ verificaram que em adolescentes com média de idade de 16,5 $\pm 1,5$ anos, a insatisfação corporal manifestou-se em 40,6\% pelo BSQ e em 45,8\% pela Escala de Silhuetas. Nota-se que houve diferença nas prevalências de meninas com insatisfação corporal, sendo que pela ES esse número foi superior. Além disso, observou-se associação entre o número de refeições e a insatisfação corporal avaliada apenas pelo BSQ. O que ocorreu, possivelmente, devido às discrepâncias entre os dois instrumentos, sendo que o BSQ é um questionário extenso e que demanda mais tempo para responde-lo, enquanto que a aplicação da ES é mais rápida e sua interpretação mais fácil. Este resultado não foi constatado pela avaliação da imagem corporal utilizando a escala de silhuetas (ES).

Verificou-se que as adolescentes realizavam 4,06 $( \pm 0,85)$ refeições por dia, durante a semana e 3,98 $( \pm 1,02)$ nos finais de semana. Recomenda-se fazer pelo menos 5 refeições por dia, para se ter uma alimentação fracionada e mais saudável. ${ }^{7}$ A maior frequência de refeições diárias pode estar relacionada a menor ocorrência de sobrepeso e obesidade em adolescentes, ${ }^{31,32}$, e, consequentemente, a menor insatisfação corporal uma vez que adolescentes com excesso de peso são mais propícias a serem insatisfeitas com seus corpos. ${ }^{28} \mathrm{Na}$ população adolescente observa-se um comportamento alimentar que inclui a omissão de refeições, principalmente o café da manhã e substituição do almoço ou jantar, por lanches, muitas vezes calóricos e associados ao consumo de refrigerantes. A substituição das principais refeições por lanches pode ser devido à maior praticidade em se consumir alimentos prontos ou semiprontos ${ }^{7}$.

Portanto, a omissão de refeições, além de contribuir para uma alimentação inadequada e o desenvolvimento de excesso de peso, também pode favorecer a 
ocorrência de insatisfação com a imagem corporal.33,34 Um estudo realizado com crianças e adolescentes mostrou que fazer mais de quatro refeições diárias foi mais prevalente em adolescentes do sexo masculino $(77,9 \%)$ em comparação com o sexo feminino $(66,6 \%)(p=0,001)$. Ainda, as meninas que realizavam menos de quatro refeições por dia tiveram 1,53 vezes mais risco de apresentar excesso de peso, quando comparadas àquelas que faziam um maior número de refeições diárias (RP $=1,53 ;$ IC $95 \%: 1,06-2,22 ; \mathrm{p}=0,022)^{10}$.

Bibiloni e colaboradores ${ }^{35}$ verificaram que a maioria $(96,6 \%)$ das adolescentes avaliadas com excesso de gordura corporal eram insatisfeitas com a imagem corporal, e, comparadas às com percentual de gordura corporal adequado, apresentaram maior prevalência de apenas três ou menos refeições realizadas diariamente $(41,7 \%$ e $60,2 \%$, respectivamente; $p=0,006)$.

Mudanças no hábito alimentar é uma das estratégias que pode ser usada para tentar melhorar o sentimento com a imagem corporal. Porém, muitas vezes estas atitudes são feitas de forma equivocada, sem orientação de um nutricionista. Os resultados desta pesquisa confirmam tais suposições ao mostrar que as adolescentes mais insatisfeitas foram aquelas com menor número de refeição semanal e uma pequena parte dessa população foi classificada com a frequência alimentar referente aos grupos de alimentos adequada. É comum mulheres adotarem práticas alimentares inadequadas para redução de peso, quando não estão satisfeitas com a própria imagem $^{2,11,30}$.

Além da quantidade, a qualidade dos alimentos também influencia no desenvolvimento de excesso de peso, e consequentemente na avaliação negativa da imagem corporal. Adolescentes em geral possuem um hábito alimentar não saudável, com elevado consumo de alimentos ultraprocessados ricos em gorduras, açúcares e sódio e baixa ingestão de alimentos ricos em fibras, vitaminas e minerais ${ }^{6,7}$. Portanto, fazer restrições no número de refeições realizadas diariamente pode não só prejudicar a saúde das adolescentes, como também não favorece a perda de peso, que é o objetivo pretendido com tais restrições. É necessário e muito importante que as adolescentes recebam orientação de um profissional qualificado, para que assim ocorra a reeducação alimentar que vai possibilitar que o indivíduo consiga alcançar uma composição corporal adequada de forma saudável, sem riscos à sua saúde ${ }^{35}$. 
A avaliação da frequência de refeições por um questionário reduzido e autoaplicável pode ser uma limitação do estudo, porém, é um questionário validado e usualmente utilizado em estudos, além disso, foi uma alternativa usada para uma avaliação prática e objetiva. Outra limitação que pode ser considerada é o delineamento transversal, que não permite avaliar a relação entre causa e efeito, de forma que impossibilita concluir se a insatisfação corporal causa a redução no número de refeições diárias ou o contrário. Entretanto, ainda assim, o estudo observou associação entre a insatisfação corporal, composição corporal e o número de refeições realizadas. Ademais, os resultados aqui observados mostram a lacuna que existe na literatura, considerando que ainda são escassos os estudos que avaliam como se dá a associação entre a imagem corporal e hábitos de vida em adolescentes. Portanto, esse é um dos poucos estudos que investigaram a relação entre a insatisfação corporal e frequência alimentar em adolescentes. Outro ponto a se considerar é o uso de dois instrumentos validados para a avaliação da insatisfação corporal nessa população, o que proporciona maior confiabilidade aos resultados.

\section{REFERÊNCIAS}

1. Tsai MC, Strong C, Lin CY. Effects of pubertal timing on deviant behaviors in Taiwan: a longitudinal analysis of 7 th- to 12th-grade adolescents. J Adolesc 2015; 42: 87-97.

2. Morais NS, Miranda VPN, Priore SE. Body image of female adolescents and its association with body composition and sedentary behavior. Cien Saude Colet 2018; 23 (8): 2693-2703.

3. Malina R, Bouchard C, Bar-Or O. Crescimento, maturação e atividade física. São Paulo: Phorte; 2009.

4. Lemes DCM, Câmara SG, Alves GG, Aerts D. Body image satisfaction and subjective wellbeing among ninth-grade students attending state schools in Canoas, Brazil. Cien Saude Colet 2018; 23(12):4289-4298.

5. Miranda VPN, Conti MA, Bastos RR, Laus MF, Almeida SS, Ferreira MEC. Imagem corporal de adolescentes de cidades rurais. Cien Saude Colet 2014; 19 (6): 17911801.

6. Vasconcellos MB, Anjos LA, Vasconcellos MTL. Estado nutricional y tiempo de pantalla de escolares de la red pública de enseñanza fundamental de Niterói, Río de Janeiro, Brasil. Cad. Saúde Pública. 2013; 29 (4): 713-722.

7. Priore SE, Morais NS, Cecon RS, Miranda VPN. Avaliação Nutricional de Adolescentes: Aspectos Clínicos e Laboratoriais. In: Almeida MFL, Capelli JCS, Sperandio N, Rocha CMM, Ribeiro BG. Alimentação e Nutrição da Infância à Adolescência. São Paulo: RED, 2018. p. 51-68.

8. Schuck K, Munsch S, Schneider S. Body image perceptions and symptoms of disturbed eating behavior among children and adolescents in Germany. Cineantropom. Child Adolesc Psychiatry Ment Health 2018; 12 (10): 1-11. 
9. Silva PO, Guimarães JMN, Griep RH, Melo ECP, Matos SMA, Molina MDC. Association between Body Image Dissatisfaction and Self-Rated Health, as Mediated by Physical Activity and Eating Habits: Structural Equation Modelling in ELSA-Brasil. Int. J. Environ. Res. Public Health 2018; 15 (4): 790.

10. Silva FA, Candiá SM, Pequeno MS, Sartorelli DS, Mendes LL, Oliveira RMS, Netto MP, Cândido APC. Frequência de refeições diárias e variáveis associadas em crianças e adolescentes. J. Pediatr. Porto Alegre 2017; 93 (1): 79-86.

11. Miranda VPN, Morais NS, Faria ER, Amorim PR, Marins JCB, Franceschini SCC, Teixeira PC, Priore SE. Body dissatisfaction, physical activity, and sedentary behavior in female adolescents. Rev Paul Pediatr 2018; 36 (4): 482-490.

12. Laus MF, Almeida SS, Murarole MB, Braga-Costa TC. Estudo de Validação e Fidedignidade de Escalas de Silhuetas Brasileiras em Adolescentes. Psic.: Teor. e Pesq. Brasília 2013; 29 (4): 403-409.

13. Conti MA, Cordás TA, Latorre MRDO. A study of the validity and reliability of the Brazilian version of the Body Shape Questionnaire (BSQ) among adolescents. Rev. Bras. Saúde Mater. Infant. Recife 2009. 9 (3): 331-338.

14. Kakeshita IS, Silva AIP, Zanatta IP, Almeida SS. Estudo de Validação e Fidedignidade de Escalas de Silhuetas Brasileiras em Adolescentes. Psic.: Teor. e Pesq. Brasília 2009; 25 (2): 263-270.

15. De Onis M, Onyango AW, Borghi E, Siyam A, Nishida C, Siekmann J. Development of a WHO growth reference for school-aged children and adolescents. Bulletin of the World Health Organization, Geneva 2007; 85: 660-7.

16. Williams DP, Going SB, Lohman TG, Harsh DW, Srinivasan SR, Webber LS, Berenson GS. Body fatness and risk for elevated blood pressure, total cholesterol, and serum lipoprotein ratios in children and adolescents. Am J Public Health 1992; 82 (3): 358-63.

17. World health organization. Waist circumferences and waist-hip ratio: report of a WHO expert consultation, Geneva, World Health Organization, 2008.

18. Pereira PF, Serrano HMS, Carvalho GQ, Lamounier JA, Peluzio MCG, Franceschini SCC, Priore SE. Circunferência da cintura e relação cinturaestatura: úteis para identificar o risco metabólico em adolescentes do sexo feminino? Rev. Paul. Pediatr. 2011; 29 (3): 372-7.

19. Priore SE. Composição corporal e hábitos alimentares de adolescentes: uma contribuição à interpretação de indicadores do estado nutricional. [PhD thesis]. 211 f. Universidade Federal de São Paulo - Escola de Medicina. São Paulo, 1998.

20. Barbosa KBF. Consumo alimentar e marcadores de risco para a síndrome metabólica em adolescentes do sexo feminino: comparação entre instrumentos de inquérito dietético. 2006. 228 p. Dissertação (Mestrado em Ciência da Nutrição) - Universidade Federal de Viçosa, Viçosa, 2006.

21. Barufaldi LA, Abreu GA, Oliveira JS, Santos DF, Fujimori E, Vasconcelos SML, et al. ERICA: prevalência de comportamentos alimentares saudáveis em adolescentes brasileiros. Rev Saúde Pública. 2016; 50 (1): 1s-9s.

22. Olatona FA, Onabanjo OO, Ugbaja RN, Nnoaham KE, Adelekan DA. Dietary habits and metabolic risk factors for non-communicable diseases in a university undergraduate population. J Health Popul Nutr. 2018; 16; 37 (1): 21. 
23. Maia EG, Silva LES, Santos MAS, Barufaldi LA, Silva SU, Claro RM. Padrões alimentares, características sociodemográficas e comportamentais entre adolescentes brasileiros. Rev Bras Epidemiol. 2018; 21 (1): E180009.

24. Rathi N, Riddel L, Worsley A. Food consumption patterns of adolescents aged 14-16 years in Kolkata, India. Nutrition journal. 2017; 16: 1-12.

25. Faria ER, Franceschini SCC, Peluzio MCG, Sant'Ana, LFR, Priore SE. Correlação entre Variáveis de Composição Corporal e Metabólica em Adolescentes do Sexo Feminino. Arq. Bras. Cardiol. 2009; 93 (2): 119-127.

26. Morais NS, Miranda VPN, Priore SE. Imagem corporal de adolescentes do sexo feminino e sua associação à composição corporal e ao comportamento sedentário. Ciênc. saúde coletiva. 2018; 23 (8): 2693-2703.

27. Ripka WL, Orsso CE, Haqq AM, Prado CM, Ulbricht L, Leite N. Validity, accuracy of body fat prediction equations using anthropometrics measurements in adolescentes. Eat Weight Disord. 2021; 26: 879-886.

28. Miranda VPN, Amorim PRS, Bastos RR, Souza VGB, Faria ER, Franceschini SCC, Priore SE. Evaluation of lifestyle of female adolescents through latent class analysis approach. BMC Public Health. 2019; 19 (184): 1-12.

29. Ribeiro-Silva RC, Fiaccone RL, Conceição-Machado MEP, Ruiz AS, Barreto ML, Santana MLP. Body image dissatisfaction and dietary patterns according to nutritional status in adolescents. J Pediatr. 2018; 94 (2): 155-161.

30. Alvarenga MS, Scagliusi FB, Phillippi ST. Comportamento de risco para transtorno alimentar em universitárias brasileiras. Rev. Psiquiatr. Clín. 2011; 38 (1): 3-7.

31. Kelishadi R, Qorbani M, Motlagh ME, Heshmat R, Ardalan G, Bahreynian M. Association of eating frequency with anthropometric indices and blood pressure in children and adolescents: the CASPIAN-IV study. J Pediatr. 2016; 92: 156-167.

32. Jäskeläinen A, Schwab U, Kolehmainen M, Pirkola J, Järvelin MRJ. Associations of meal frequency and breakfast with obesity and metabolic syndrome traits in adolescents of Northern Finland Birth Cohort 1986. Nutr Metab Cardiovasc Dis. 2013; 23: 1002-1009.

33. Estima CC, Costa RS, Sichieri R, Pereira RA, Veiga GV. Meal consumption patterns and anthropometric measurements in adolescents from a low socioeconomic neighborhood in the metropolitan area of Rio de Janeiro, Brazil. Appetite. 2009; 52: 735-739.

34. Leal GV, Philippi ST, Matsudo SM, Toassa EC. Consumo alimentar e padrão de refeições de adolescentes, São Paulo, Brasil. Rev Bras Epidemiol. 2010; 13: 457467.

35. Bibloni M del M, Pich J, Pons A, Tur JA. Body image and eating patterns among adolescents. BMC Public Health. 2013; 13: 1104.

Submissão: 05/02/2020

Aprovação: 29/06/2021 